\title{
Le Pascal de la joie et de la félicité
}

Hommage à Philippe Sellier

\section{Maria Vita Romeo}

\section{CpenEdition}

\section{Journals}

Édition électronique

URL : http://journals.openedition.org/ccibp/315

DOI : $10.4000 /$ ccibp.315

ISSN : 2493-7460

Éditeur

Centre international Blaise Pascal

\section{Édition imprimée}

Date de publication : 5 février 2014

Pagination : 24-26

ISBN : 978-2-84516-693-6

ISSN : 0249-6674

Référence électronique

Maria Vita Romeo, "Le Pascal de la joie et de la félicité », Courrier du Centre international Blaise Pascal [En ligne], 36 | 2014, mis en ligne le 03 décembre 2015, consulté le 15 septembre 2020. URL : http:// journals.openedition.org/ccibp/315

Ce document a été généré automatiquement le 15 septembre 2020.

Centre international Blaise Pascal 


\title{
Le Pascal de la joie et de la félicité
}

\author{
Hommage à Philippe Sellier
}

\author{
Maria Vita Romeo
}

1 La traduction par Maria Vita Roméo du recueil d'études de Philippe Sellier, Pascal et Port-Royal, a donné lieu le 21 janvier à un hommage qui s'est tenu à Catane, depuis plusieurs années haut lieu des études pascaliennes. Un séminaire s'est tenu autour de l'actualité de Pascal, au cours duquel ont pris la parole Giuseppe Pezzino, Roberto Osculati, et Antonio Paese.

2 Mardi 21 janvier 2014, dans la prestigieuse salle du «Coro di Notte » du monastère des Bénédictines, Université de Catane, a été présentée l'œuvre de Philippe Sellier, Pascal e Port-Royal (Éditeur Morcelliana, Brescia 2013, p. 620), éd. par Maria Vita Romeo (Université de Catane). Par cette traduction italienne de l'œuvre de Philippe Sellier, Maria Vita Romeo atteint significativement une étape ultérieure de son activité de recherche, en mettant à disposition des spécialistes italiens une autre œuvre précieuse dans le domaine des études pascaliennes. En effet, après la traduction italienne de l'œuvre de Jean Mesnard, Sui «Pensieri» di Pascal (éditeur Morcelliana, Brescia 2011), maintenant $\mathrm{M}^{\text {me }}$ Romeo présente aux savants et aux spécialistes italiens une autre œuvre capitale sur le Clermontois, en introduisant ainsi en Italie les travaux des deux plus grands spécialistes actuels au niveau mondial d'études pascaliennes : Jean Mesnard et Philippe Sellier. Par ces deux opérations culturelles, on peut dire qu'en deux ans environ, elle a fourni des instruments de recherche scientifique utiles pour une connaissance à ronde-bosse de Blaise Pascal et de son entourage. Maintenant, en effet, le Pascal savant, philosophe et mystique de J. Mesnard est admirablement complété par le Pascal spirituel et théologien ciselé magistralement par M. Sellier qui, non seulement éclaire l'« énigme Port-Royal » - c'est-à-dire le phénomène très rare d'un groupe restreint des personnes qui exercent un poids considérable sur la culture du XVII ${ }^{e}$ siècle français et européen -, mais il nous offre aussi un Pascal penseur très « actuel ", qui traite merveilleusement des problèmes qui ne sont pas éloignés de la sensibilité culturelle qui émergera dans le $\mathrm{XX}^{\mathrm{e}}$ siècle avec la crise de la modernité, avec l'avènement des totalitarismes et avec la tragédie historique de la seconde guerre mondiale. 
Par ces deux classiques de la littérature critique pascalienne - lesquels, grâce à $\mathrm{M}^{\mathrm{me}}$ Romeo, occupent maintenant une grande place dans le panorama culturel italien - la figure d'un Pascal auteur des Provinciales et des Pensées (traditionnellement très chère aux spécialistes italiens) se complète par la figure d'un Pascal savant, théologien, philosophe et surtout d'un Pascal spécialiste de l'homme et de Dieu. Autrement dit, d'un Pascal qui étudie l'homme pour souligner non seulement sa misère, mais aussi sa grandeur, afin de parvenir à la félicité et à la réalisation d'une éthique conforme à l'enseignement de la religion chrétienne fondée sur la joie.

4 Dans la Prière pour demander à Dieu le bon usage des maladies - une sorte de poème en prose -, Pascal chante les délices et le bonheur que Dieu donne à l'âme : « 0 mon Dieu ! Qu'une âme est heureuse dont vous êtes les délices, puisqu'elle peut s'abandonner à vous aimer, non seulement sans scrupule, mais encore avec mérite! Que son bonheur est ferme et durable... Oh! Qu'heureux sont ceux qui avec une liberté entière et une pente invincible de leur volonté aiment parfaitement et librement ce qu'ils sont obligés d'aimer nécessairement !» En fait, on peut affirmer que la philosophie pascalienne (au sens large du mot) est la "philosophie de la joie", parce qu'il a compris à fond le vrai objectif de la philosophie : c'est-à-dire indiquer à l'homme comment parvenir à la félicité par la compréhension de la misère et de la grandeur, ou mieux par la compréhension de la coincidentia oppositorum entre misère et grandeur, qui permettra à l'homme de s'estimer à sa juste valeur et, par conséquent, de s'ouvrir aux autres et à Dieu en détruisant sa carapace d'amour de soi. De cette manière, l'homme pourra parvenir à la vraie morale, sur l'indication du fragment des «membres pensants » et agir pour le bien commun, comme on enseignait dans les murs de Port-Royal, parce que le «bien agir » est directement lié au "bien penser ». En un certain sens, on peut dire que, pour Pascal, la connaissance scientifique, quoiqu'elle soit autonome dans ses valeurs et dans ses procédés, est toutefois propédeutique à l'agir moral. On pense par exemple à l'opuscule De l'esprit géométrique, où Pascal affirme que par l'application de la méthode géométrique l'homme arrive à former des réflexions « qui valent mieux que tout le reste de la géométrie même ».

D'autre part, la présentation de ce volume a été pensée aussi comme un véritable « hommage à Sellier » pour lequel, comme il déclare lui-même dans la Préface italienne, Pascal est un penseur qui l'a accompagné toute sa vie. Pour Sellier, en effet, Pascal a été " infiniment plus qu'un objet d'étude "; il a été un compagnon de voyage qui l'a aidé à s'orienter entre les instances de l'existentialisme moderne et les leçons éternelles de saint Augustin. Pascal, un ami qui lui a dévoilé les secrets pour saisir le sens du Deus absconditus, dont Pascal fit expérience la nuit du 23 novembre 1654. Dans le fameux Mémorial, Pascal nous rappelle que la foi est «certitude, certitude, sentiment, joie, paix ». Bien inspiré «directement» de Pascal, Sellier nous mène par la main dans l'univers pascalien au moyen de différentes lectures interprétatives sur les thèmes de la misère et de la grandeur, de la prophétie, de la théorie des trois ordres, qui impose aux yeux du cœur la personnalité mystérieuse de Jésus-Christ porteur de cette joie, laquelle n'a jamais abandonné Pascal. Coordonnés par $\mathrm{M}^{\mathrm{me}}$ Romeo, trois "amis de Pascal», spécialistes de la pensée philosophique du XVII ${ }^{e}$ siècle, ont présenté le livre italien de Philippe Sellier: Paolo Amodia (Université de Naple), Domenico Bosco (Université Chieti-Pescara) et Carmelo Vigna (Université de Venice).

6 Paolo Amodia souligne la "méthode de Sellier ", qui nous surprend quand il nous présente un Pascal actuel qui parle à chacun d'entre nous en fin connaisseur des 
problématiques d'aujourd'hui. Bref, un Pascal qui dialogue avec Nathalie Sarraute, sans oublier Montaigne ni le maître Augustin ; un Pascal qui « dicte les temps de la scansion de l'entendre mystique », quoique autrement que saint Jean de la Croix ou sainte Teresa d'Avila.

Domenico Bosco saisit tout de suite la grande leçon de Philippe Sellier, selon lequel pour comprendre Pascal et son entourage il faut «en finir avec deux préjugés au moins : la tristesse janséniste et Port-Royal anti-mystique »; d'où la nécessité de mettre en lumière le Pascal spirituel au cœur plein de joie, qui se met en prière pour remercier Dieu de se montrer à l'âme. De cette manière, on comprend l'importance du Mémorial que Bosco définit comme un véritable chant : un chant pour le Dieu qui se rencontre dans l'Évangile, un Dieu qui donne joie et qui console, un Dieu qui met en relation avec l'autre, selon la morale chrétienne qui se fonde sur le rapport inséparable entre les parties et le tout ! Et le Dieu qui donne la joie est sans aucun doute le Dieu que Pascal apprend à connaître par saint Augustin. Aussi, quand Pascal écrivait les Pensées, il prenait comme modèle le De vera religione de saint Augustin, un livre que Saint-Cyran conseillait aux débutants et qu'Arnauld avait traduit en 1647. D'où le projet pascalien de rédiger une apologie qui entendait se rapprocher du christianisme avec la douceur, pour rendre la « religion aimable». Voilà le Pascal champion de la joie chrétienne, fruit fécond d'un "prophétisme » qui vient par la grâce et qui travaille pour la grâce ; voilà le Pascal chrétien et heureux, que Sellier a magistralement présenté au lecteur, en tressant habilement saint Augustin, Pascal et Port-Royal. Philippe Sellier donc nous porte aussi à l'intérieur de l'univers des Pensées par les Confessions de saint Augustin, en tenant toujours un œil fixé sur la Bible, et en particulier le Psaume 118, et sur l'Évangile de saint Jean, lesquels, pour Pascal, sont des points fixes dont quotidiennement il tire inspiration et enseignements. D'où le triomphe d'un Pascal mystique qui aime JésusChrist en tant que source unique de joie et de félicité.

Carmelo Vigna met en évidence l'importance du projet apologétique par lequel le Clermontois entend dialoguer avec l'athée moderne, enclin à l'indifférence. D'où l'analyse de la misère humaine, qui trouve une justification dans la nécessité de montrer à l'homme moderne toute sa fragilité sans le Dieu des chrétiens, porteur du vrai bonheur.

Dans le cadre de l'« hommage à Philippe Sellier ", les organisateurs ont ensuite pensé aussi à un séminaire sur l'actualité de Pascal, avec l'intervention de trois professeurs de l'Université de Catane : Roberto Osculati, Antonio Pesce et Giuseppe Pezzino.

Roberto Osculati, historien du christianisme, parle de la spiritualité pascalienne brillamment mise en lumière par Philippe Sellier, une spiritualité qui non seulement fait ses comptes avec la modernité, mais qui entre dans la postmodernité tout de suite, en y trouvant une place d'honneur. De cette manière, coexistent le Pascal ultramoderne, le Pascal de Baudelaire, de Nathalie Sarraute ou de Valéry, qui se mêlent au Pascal des colorations oratoriennes riches de spiritualité chrétienne.

11 Antonio Pesce, pour sa part, concentre sa réflexion sur le rapport entre Pascal et Mounier qui, ayant vécu une époque de crise profonde, s'adresse à Pascal, qui parle toutes les langues de toutes les époques parce qu'il connaît au fond le cœur de l'homme. On passe ainsi d'un Pascal existentialiste à un Pascal métaphysique, qui se passionne tant à la pensée qu'à l'être, qui sait concilier le monde et l'intériorité comme seulement un métaphysicien du « visage humain » sait le faire. 
iuseppe Pezzino enfin parle d'un Pascal qui peut être compté non seulement entre les grands esprits de la pensée philosophique, pour son effrayant génie, mais aussi parce que «Pascal est l'ami dans lequel on peut trouver refuge ; il est l'ami qui te tient compagnie et qui te connaît parce qu'il accède à la conscience. Blaise est l'un d'entre nous! ». Or, pour celui qui connaît M. Pezzino, sa philosophie, sa mentalité dialectique qui l'« empêche » d'exalter dogmatiquement et unilatéralement un auteur ou une pensée philosophique, ses mots ont un goût spécial. C'est la fascination de Pascal qui pénètre aussi l'esprit des philosophes les plus prudents.

Ainsi, M. Pezzino fait ses comptes avec la raison pascalienne en employant les «raisons» de Philippe Sellier, qui a indiqué une sorte de "Critique de la raison pascalienne ». Bref, il fait ses comptes aussi avec la-philosophie pascalienne qui se moque du stoïcisme et du scepticisme, où la raison devient faible, paralysée, piégée par le divertissement, qui fuit le relativisme pour aborder au bonheur.

Sans aucun doute cette journée d'études légitime et consolide ultérieurement le rôle du Centre d'études pascaliennes de l'Université de Catane, qui collabore avec le Centre international Blaise Pascal de Clermont-Ferrand et avec le Centre d'étude de la langue et de la littérature françaises du XVII ${ }^{e}$ et XVIII ${ }^{e}$ siècle de Paris-Sorbonne. Pendant cette journée a émergé le Pascal de la joie et de la certitude, dont la pensée a un impératif : poursuivre le bonheur, c'est-à-dire le souverain bien. Un bonheur qui restera dans la sphère du temps, si l'âme de l'homme n'est pas touchée par la grâce, l'unique moyen qui donne la foi en permettant ainsi à l'homme de jouir du bonheur éternel, qui consiste dans la réconciliation avec Dieu.

Se réconcilier avec Dieu signifie ouvrir le cœur à Dieu, en méprisant le moi, c'est-à-dire l'égoïsme dicté par l'amour propre. De cette manière on peut commencer à faire les premiers pas pour être heureux et édifier la vraie morale, fondée simplement sur la charité évangélique dont le modèle a été merveilleusement synthétisé par Pascal dans la liasse intitulée « Morale chrétienne ». À ce propos est très significatif le fragment des Pensées où on lit : «Pour régler l'amour qu'on se doit à soi-même, il faut s'imaginer un corps plein de membres pensants, car nous sommes membres du tout, et voir comme chaque membre devrait s'aimer " (401 Sel.). De cette manière, il est possible parvenir à la félicité, parce que «nul n'est heureux comme un vrai chrétien, ni raisonnable, ni vertueux, ni aimable » (389 Sel.). 
Affiche de la journée d'étude "Éthique et théologie à Port-Royal". Hommage à Philippe Sellier. 21 janvier 2014

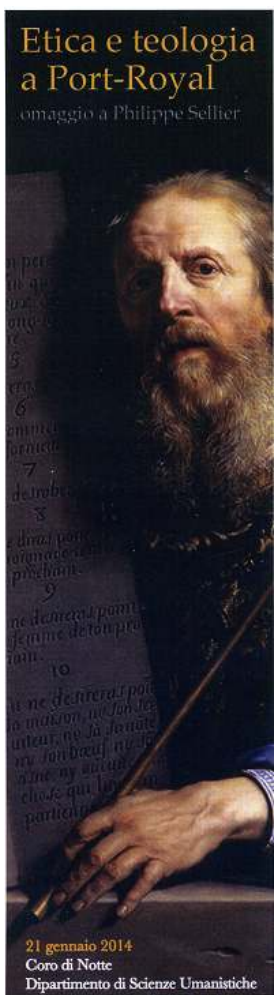

Département des sciences humaines. Université de Catane. Italie

INDEX

Mots-clés : Pascal, joie, félicité

Keywords : Pascal, joy, bliss

\section{AUTEUR}

\section{MARIA VITA ROMEO}

Centre d'Études de la Langue et de la Littérature Françaises des XVIIe et XVIIIe siècles, Université Paris Sorbonne 